\title{
Protein inhibitor of activated STAT-1 is downregulated in gastric cancer tissue and involved in cell metastasis
}

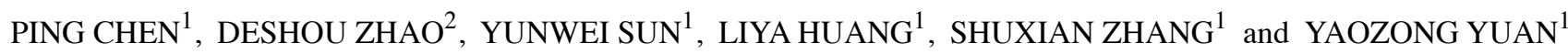 \\ ${ }^{1}$ Department of Gastroenterology, Ruijin Hospital, Shanghai Jiaotong University School of Medicine, Shanghai 200025; \\ ${ }^{2}$ Department of Laboratory, Second Hospital Affiliated Lanzhou University, Lanzhou, Gansu 746000, P.R. China
}

Received April 30, 2012; Accepted July 28, 2012

DOI: 10.3892/or.2012.2030

\begin{abstract}
Protein inhibitor of activated STAT-1 (PIAS1) is a novel modulator of the JAK/STAT signaling pathway that negatively regulates the inflammatory response. It has been also reported to be downregulated in a variety of human cancer cell lines. However, the role of PIAS1 in gastric cancer remains unclear. In this study, we investigated the prognostic value of PIAS1 expression and its regulated mechanisms in gastric cancer cell metastasis. Therefore, the expression of PIASI was explored in gastric cancer tissues and adjacent tissues of gastric cancer with 31 cases of patients, and the prognostic value was analyzed. In addition, the growth and invasion in SGC7901 cells were investigated in the restoration of PIAS1 expression with Ad5/F35-PIAS1 or Ad5/F35-vector or PBS treatment, and the activity of P38MAPK, P-P38MAPK, JNK/SAPK, P-JNK/SAPK, ERK and P-ERK, were detected by western blotting. The tumor migratory factors MMP-9, MMP-2 and ICAM-1 were analyzed by western blotting. The results demonstrated that 22 of 31 (70.9\%) gastric cancer specimens showed low levels of PIAS1 expression from immunohistochemistry staining using tissue microarrays. Statistical analysis suggested that the downregulation of PIAS1 was significantly correlated with tumor staging. Furthermore, we found that the restoration of PIAS1 expression mediated by Ad5/F35 virus suppressed cell proliferation and invasion accompanied by the inhibition of P38MAPK and ERK protein expression and activity, but not JNK/SAPK protein. Notably, PIAS1 restoration with the transfection of Ad5/F35-PIAS1 robustly decreased the expression of tumor migratory factors including MMP-9, MMP-2 and ICAM-1 compared to Ad5/ F35-vector. These data suggest that PIAS1 may function as a tumor suppressor to regulate gastric cancer cell metastasis by targeting the MAPK signaling pathway.
\end{abstract}

Correspondence to: Dr Yaozong Yuan, Department of Gastroenterology, Ruijin Hospital, Shanghai Jiaotong University School of Medicine, Shanghai 200025, P.R. China

E-mail: yuanyz28@yahoo.com.cn

Key words: protein inhibitor of activated STAT-1, gastric cancer, MAPK signaling pathway, migration

\section{Introduction}

Gastric cancer is still a major health problem and a leading cause of cancer-related death although its incidence is decreased worldwide (1). In spite of the standardization of surgical techniques and multimodal therapy, the postoperative survival of patients with advanced gastric cancer still remains very low. It was recently reported that the understanding of the molecular and genetic factors involved in gastric cancer progression may identify novel gastric biomarkers and highlight potential avenues of investigation for targeted therapies.

Recent evidence shows that PIAS1, a downstream target protein of STAT signaling pathway inhibitor, is related to antiinflammatory response through negative regulation of NF-kB/ STAT1 signaling, which mediates inflammatory cell adhesion and inhibits inflammatory injury (2). Consistently, PIAS1 null mice show increased protection against pathogenic infection and are hypersensitive to LPS-induced septic shock (3). This finding provides a theoretical basis for revealing the mechanism of reversing inflammation by PIAS1. However, recent studies demonstrated that the expression of PIAS1 in some cancer cells is significantly downregulated or lost (4). Several studies showed that PIAS1 negatively regulates $\mathrm{Bcl}_{2}$ expression and thus reverses the cancer growth. Therefore, PIAS1 not only plays functions of inflammatory inhibitor but also plays a role in the reversal of cancer cell growth (4). Therefore, in this study, the PIAS1 expression was first observed the expression of gastric cancer tissue with patient to prove that PIAS1 may be involved in the pathogenesis of cancer and to verify whether PIAS1 acts as a marker for the preclinical detection and clinical assessment of patients with gastric cancer.

The cascade reaction of mitogen-activated protein kinases (MAPKs) is one of the vital intracellular signal transduction systems, participating in many physiological progressions, such as cell growth, proliferation, differentiation and apoptosis. Three major subclasses of MAPKs, namely, extracellular signalregulated kinase (ERK), c-Jun NH2-terminal kinase (JNK), and P38MAPK have been identified (5). Related studies indicated that MAPKs are proposed to be a critical integrator of various signaling transduction systems and involved in various cellular processes including cell proliferation, differentiation, apoptosis, and transformation (6). Constitutive activation of these signaling cascades has been noted in the malignant transformation of 
various cell lines and implicated in carcinogenesis and metastatic potential of human cancers (7).

Therefore, we hypothesize that the regulation of PIAS1 is a key step in the multiple biological regulations in gastric cancer by regulating the MAPK signaling pathway. To test this hypothesis, we used gastric cancer SGC7901 cells and studied the migration response of the cells by upregulating administration of PIAS1. Additionally, to gain a better understanding of the mechanism of action of PIAS1, this study investigated further the effect of PIAS1 on the levels of the MAPK signaling pathway, tumor migratory factors including MMP-9, MMP-2, and ICAM-1, then providing a possible effective mechanism for gastric cancer treatment.

\section{Materials and methods}

\section{Reagents}

Patients and tissue samples. Tumor samples were obtained from gastric cancer patients who underwent surgery between 2002 and 2010 at RuiJin Hospital (Shanghai Jiaotong University School of Medicine, Shanghai, China). All cases were staged according the guidelines of the International Union Against Cancer (2002). There were 18 males and 13 females with ages ranging from 20 to 92 years (mean 60.9 years). All available clinicopathological data were collected (Table I). The study was approved by the ethical review board of Ruijin Hospital.

Tissue microarray construction and immunohistochemistry. Formalin-fixed paraffin-embedded samples were reviewed for tissue array construction by a pathologist. At least two core tissue biopsies were taken from morphologically representative regions of each gastric tumor. The samples of 31 primary tumor cases and 31 adjacent normal tissues samples were arranged in rows and columns to construct a tissue microarray. For staining, the sections $(4 \mu \mathrm{m})$ were transferred to glass slides using an adhesive slide system (PSA-CS 4; Instrumedics, Hackensack, NJ, USA) to support cohesion of the array elements. The slides were de-waxed in xylene, and rehydrated in ethanol with descending concentration. Anti-PIAS1 antibody (Santa Cruz Biotechnology, Santa Cruz, CA, USA, diluted to 1:50) was added after blocking of endogenous peroxidase and proteins, and each section was incubated with HRP-labeled anti-goat IgG antibody. The immunostained specimens were assessed by two independent observers without prior knowledge of the clinicopathologic characteristics. PIAS1 expression was scored using two measures: staining intensity was graded as 0 (negative), 1 (weak), 2 (moderate), or 3 (strong); the percentage of positive cells was scored as 0 (negative), $1(<10 \%), 2(11-50 \%)$, $3(51-80 \%)$, or $4(>80 \%)$. The two scores were multiplied and two categories of expression levels were set up: negative expression (meaning 0-2); and positive expression (meaning 3-12).

Construction of expression plasmid. We used replicationdefective adenovirus serotype 5/F35 (Ad5/F35) as the vector. Ad5/F35-PIAS1 was constructed by Benyuanzhengyang Bio. (Shanghai, China) using the previously described method (8). PIAS1 cDNA, containing the full-length translated regions, were sub-cloned into the PDC316-MCMV-EGFP transfer plasmid. This plasmid was cotransfected into 293 cells, along with a fragment of the plasmid containing the Ad5/F35 adenoviral vector.
Additionally, an Ad5/F35 containing an empty expression cassette was constructed for use as a control (Ad5/F35-vector). All of the viral constructs were similar with the exception of the trans-gene, and the production and purification procedures were identical.

Cell culture and gene transduction. The culture of human gastric cancer cell SGC7901 cells (American Type Culture Collection, Rockville, MD, USA) was conducted in the medium, containing RPMI-1640 medium (Gibco BRL, Gaithersburg, MD, USA) and 10\% fetal bovine (FBS, head-inactivated; Gibco BRL) in the ratio 1:1 and with addition of $100 \mathrm{U} / \mathrm{ml}$ penicillin and $100 \mu \mathrm{g} / \mathrm{ml}$ streptomycin (Sigma, St. Louis, MO, USA) in the standard condition $\left(37^{\circ} \mathrm{C}\right.$ and $\left.5 \% \mathrm{CO}_{2}\right)$.

In the study, when the SGC7901 cells reached $70 \%$ confluence, the transfection of Ad5/F35-PIAS1 process began as Ad5/F35-PIAS1 treated cells (MOI 10). Other SGC-7901 cells were also transfected with the empty Ad5/ F35 vector (MOI 10) (Ad5/F35-vector) as Ad5/F35 vector treated cells. Cells with PBS treatment serving as control cells. The samples were harvested at 24 or $72 \mathrm{~h}$ after the onset of induction for further experiments.

Cell viability assay and PIAS1 expression. A total of $2 \times 10^{3}$ SGC7901 cells were seeded in a 96-well plate. After $24 \mathrm{~h}$, the cells were treated with Ad5/F35-PIAS1 (MOI 10). These cells were transfected with the empty Ad5/F35 vector (MOI 10). After 24 and $72 \mathrm{~h}, 20 \mu \mathrm{l}$ 3-(4,5-dimethylthiazolyl-2)-2,5diphenyltetrazolium bromide (MTT) $(5 \mathrm{mg} / \mathrm{ml})$ was added to each well. Four hours later, $100 \mu 1$ of dimethyl sulfoxide was added to each well after the medium was removed. Finally, the absorbance was detected with an enzyme calibrator at $570 \mathrm{~nm}$ and the cell viability $=(\mathrm{A}$ of study group $/ \mathrm{A}$ of control group $)$ $\mathrm{x} 100 \%$.

PIAS1 expression in cells by RT-PCR. Total RNA was isolated from cells using TRIzol reagent kits (Gibco BRL, Rockville, $\mathrm{MD}, \mathrm{USA})$. The cDNA obtained from this reaction was mixed with PCR buffer, $\mathrm{MgCl}_{2}$, dNTPs, Taq DNA polymerase, and human PIAS1 gene-specific primers (the primer sequence of PIAS1, the forward primer: 5'-CCA CGC CTT CCT GCT GTA GA-3'; the reverse primer: 5'-TAT CAC ACA GGC AGT CTT AGA T-3') and amplified in an automated thermal cycler. The conditions of RT-PCR were as follows: 1 cycle for $5 \mathrm{~min}$ at $95^{\circ} \mathrm{C}$; 35 cycles for $45 \mathrm{sec}$ at $94^{\circ} \mathrm{C}$, for $45 \mathrm{sec}$ at $55^{\circ} \mathrm{C}$, for $1 \mathrm{~min}$ at $72^{\circ} \mathrm{C}$; 1 cycle for $10 \mathrm{~min}$ at $72^{\circ} \mathrm{C}$. The PCR products were separated by electrophoresis in $1.2 \%$ agarose gels and stained with ethidium bromide. The densities of cDNA bands were analyzed by scanning densitometry using GelDoc 2000 (Bio-Rad, Baltimore, MD, USA). The band densities were normalized to GAPDH (the primer sequence of GAPDH, the forward primer: 5'-GGC TGA GAA CGG GAA GCT TGT C-3'; the reverse primer: 5'-CAG CCT TCT CCA TGG TGG TGA AGA-3') band densities and the results were expressed as ratio.

Scratch wound-healing assay. To measure cell motility, $4 \times 10^{5}$ cells were seeded in 6-well plates. A central linear wound was created by scraping the cell monolayer with a $200-\mu 1$ sterile pipette tip. The media were carefully changed to remove any floating cells and cultured at $5 \% \mathrm{CO}_{2}$ and $37^{\circ} \mathrm{C}$. The migration of 
cells into the denuded areas in the scraped region was calculated at $48 \mathrm{~h}$, respectively. The wound at $0 \mathrm{~h}$ was considered $100 \%$ of the average gap.

Cell invasion. For the determination of invading cells, the transwell chambers (Corning Inc., NY) were used. The transwell chamber membranes were covered with $75 \mu 1$ Matrigel $^{\mathrm{TM}}$ (2 $\mathrm{mg} / \mathrm{ml}, \mathrm{BD}$ Biosciences, NJ, USA) and incubated for $2 \mathrm{~h}$ at $37^{\circ} \mathrm{C}$. The cell lines were grown in RPMI-1640 (10\% FBS) medium, thus were trypsinized and suspended at a density of $1 \times 10^{6} \%$ $\mathrm{ml}$ in serum-free RPMI-1640. Suspended cells (200 $\mu \mathrm{l})$ were placed into the upper compartment of the Transwell chambers. The lower compartment of the chambers was filled with 600 $\mu 1$ RPMI-1640 containing 10\% FBS. The transwell chamber systems were incubated in the humidified incubator $\left(37^{\circ} \mathrm{C}, 5 \%\right.$ $\mathrm{CO}_{2}$ ) for $24 \mathrm{~h}$. After incubation, the non-invaded cells and the matrigel were removed with a cotton toothpick at the top of the chamber. At the bottom of the cell swab, the invaded cells were washed with PBS and fixed with $4 \%$ paraformaldehyde and then were stained with Giemsa for $15 \mathrm{~min}$. Five random fields were counted and the invasion rate was calculated to find the number of the invaded cells under the inverted microscope at magnification $\mathrm{x} 200$. The data representing the average cells of five fields was compared between the experimental groups and control group. Each experiment was repeated three times.

Western blot analysis. The levels of P38MAPK, P-P38MAPK, JNK/SAPK, P-JNK/SAPK, ERK, P-ERK, MMP-9, MMP-2, and ICAM-1 proteins were investigated in each group using western blot analysis. The cells were washed twice with PBS and then homogenized in RIPA buffer. Following centrifugation at $12,000 \mathrm{~g}$ at $4^{\circ} \mathrm{C}$ for $10 \mathrm{~min}$, the supernatant was collected and stored at $-80^{\circ} \mathrm{C}$. Protein concentration of each sample was determined by BCA protein assay. Each sample was adjusted up to a desired protein content of $40 \mu \mathrm{g}$, thus denatured in loading buffer and separated by electrophoresis on 9\% SDS polyacrylamide gel at $100 \mathrm{~V}$ for $120 \mathrm{~min}$. The separated proteins were transferred to polyvinylidene difluoride membrane by using transfer buffer at $200 \mathrm{~mA}$ for $90 \mathrm{~min}$. The membranes were blocked with 5\% non-fat dry milk in Tris-buffered saline (TBS)- $0.1 \%$ Tween for $1 \mathrm{~h}$ at room temperature, washed three times for 10 min each in TBS- $0.1 \%$ Tween, and incubated with a primary antibody including P38MAPK, P-P38MAPK, JNK/ SAPK, P-JNK/SAPK, ERK, P-ERK, MMP-9, MMP-2, and ICAM-1 with 1:1000 dilution in TBS-0.1\% Tween overnight at $4^{\circ} \mathrm{C}$, respectively. After three $10 \mathrm{~min}$ washes in TBS- $0.1 \%$ Tween, the membranes were incubated with a second antibody, horseradish peroxidase-conjugated goat anti-rabbit or rabbit anti-mouse immunoglobulin G (Kangcheng Inc., Shanghai, China) for $1 \mathrm{~h}$ at room temperature. After washing, the membranes were detected by the enhanced chemiluminescence methods (Amersham Biosciences, Piscataway, NJ, USA), and then scanned by densitometry using bio-image analysis system (Bio-Rad) for quantification. GAPDH was determined in the similar manner to anti-GAPDH antibody (diluted to 1:500, Santa Cruz) as an endogenous control for other proteins.

Statistical analysis. All data were expressed as the mean \pm standard deviation (SD). Statistics were done by SPSS program at 10.5 versions. The $\chi^{2}$ test was used to evaluate the correlation
Table I. Relationship of PIAS1 expression with clinicopathological parameters in gastric cancer with patients.

\begin{tabular}{lccc}
\hline & Total & Positive & P-value \\
\hline Total cases & 31 & & \\
Age (years) & & & \\
High $(>50)$ & 25 & 11 & 0.318 \\
Low $(<50)$ & 6 & 4 & \\
Gender & & 5 & 0.524 \\
Female & 13 & 9 & \\
Male & 18 & & 0.00 \\
Tumor extent & & 8 & \\
T2 & 10 & 6 & 0.971 \\
T3, T4 & 21 & & \\
Size $(\mathrm{cm})$ & & 3 & \\
Large $(>5)$ & 24 & 10 & \\
Small $(<5)$ & 7 & &
\end{tabular}

between PIAS1 expression and clinicopathological characteristics. The one-way analysis of variance (ANOVA) with Dunnett's multiple comparison tests was used for comparisons. A $\mathrm{P}<0.05$ was considered as statistically significant.

\section{Results}

Expression of PIAS1 in gastric cancer tissue. We investigated the expression of PIAS1 in 31 cases of gastric cancer tissues and 31 cases of adjacent normal tissues of gastric cancer (Fig. 1). The results showed that PIAS1 levels were significantly lower in gastric cancer than in the adjacent normal tissues of gastric cancer in all 31 cases of patients. These results suggest that PIAS1 expression was significantly decreased in human gastric cancer.

Furthermore, the clinical relevance was confirmed by the observation that PIAS1 expression correlated with prognosis (Table I). Tumor staging was significantly associated with low expression of PIAS1 protein $(\mathrm{P}<0.05)$. However, a non-significant correlation was observed between PIAS1 protein expression and age with patients. No correlation was observed between PIAS1 protein expression and tumor size with patients or between PIAS1 protein expression and gender with patients $(\mathrm{P}>0.05)$.

Expression of PIAS1 in gastric cancer cells. We monitored the levels of PIAS1 transcripts in SGC7901 cells by RT-PCR assays (Fig. 2B). The data showed that at $6 \mathrm{~h}$ following Ad5/F35-PIAS1 treatment, PIAS1 mRNA levels were increased in SGC7901 cells but not in the cells with Ad5/F35-vector treatment. The enhanced PIAS1 gene expression returned to basal level within $72 \mathrm{~h}$ after stimulation. The protein expression of PIAS1 was monitored using western blot analysis (Fig. 2A). Similar to the RT-PCR data, the SGC7901 cells with Ad5/F35-PIAS1 treatment expressed a higher level of PIAS1 protein compared with SGC7901 cells with Ad5/F35-vector treatment. SGC7901 cells at $72 \mathrm{~h}$ of Ad5/F35-PIAS1 treatment did not show significant 

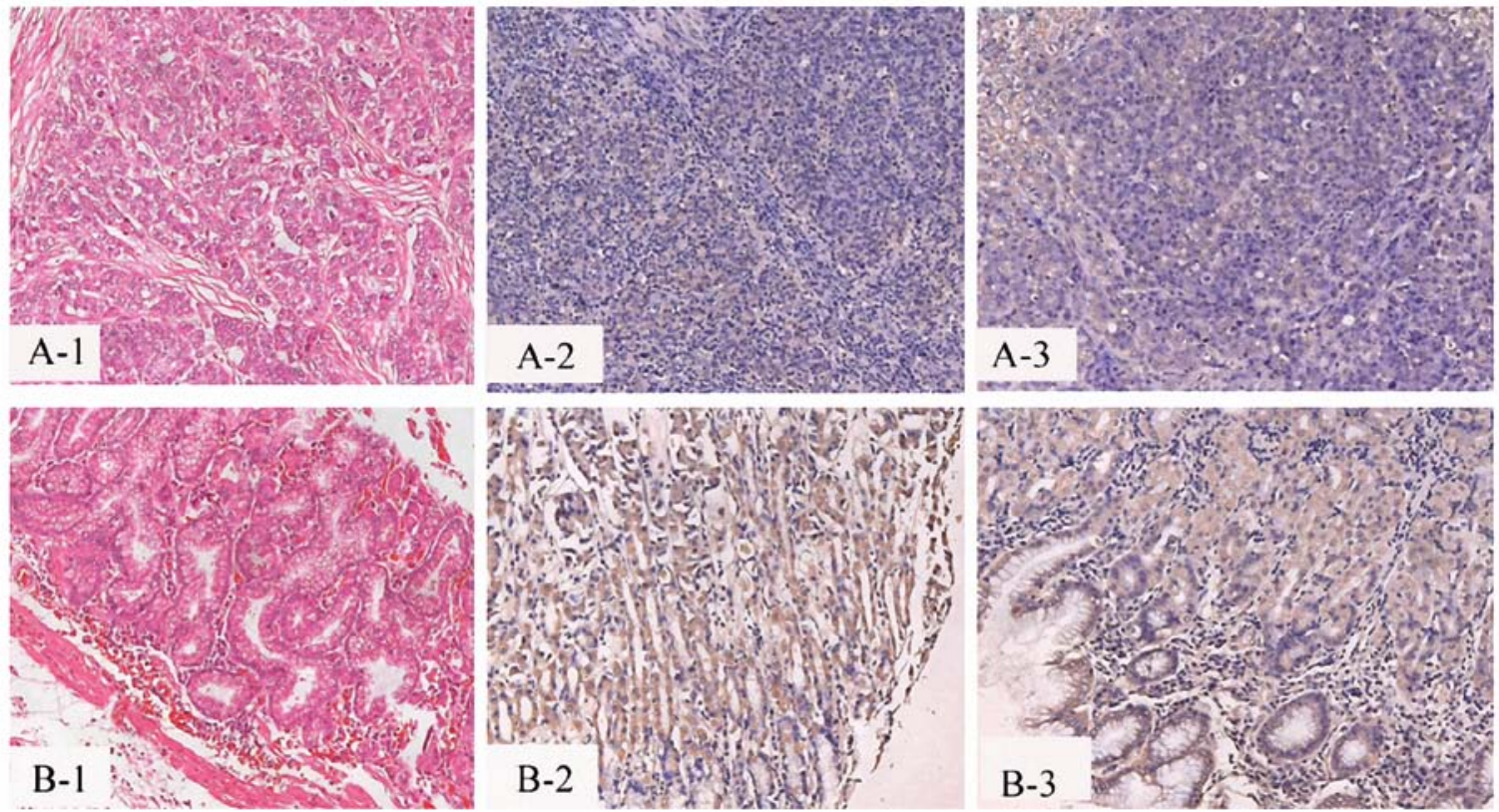

Figure 1. The evaluation of PIAS1 expression investigated in gastric cancers (A1) and adjacent normal tissues of gastric cancer (B1) on tissue microarrays by hematoxylin-eosin staining and immunohistochemical method. The invasive gastric cancers are shown with negative and weak PIAS1 staining (A2-3). The adjacent normal tissues of gastric cancer showing strong PIAS1 staining of epithelial and acinar cells (B2-3). Magnification (A1-3, B1-3) x200.

A-1

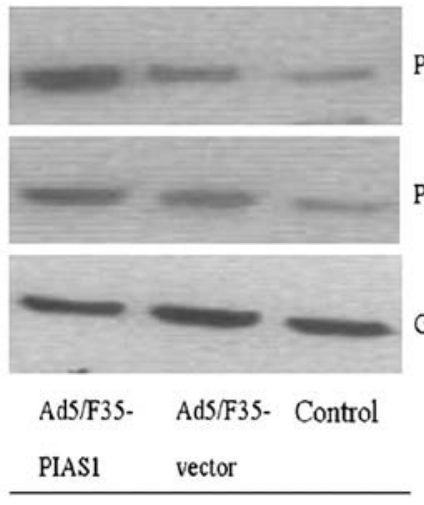

SGC7901

B-1

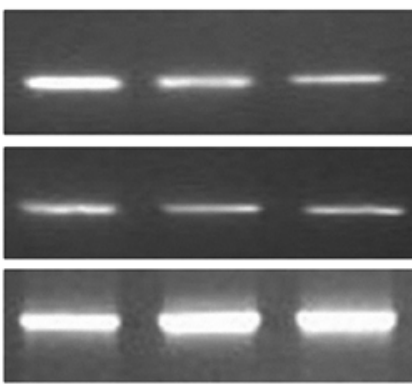

PIASl (642 bp) $24 \mathrm{~h}$

Ad5/F35- Ad5/F35- Control

PIAS1 vector

SGC7901

\section{A-2}

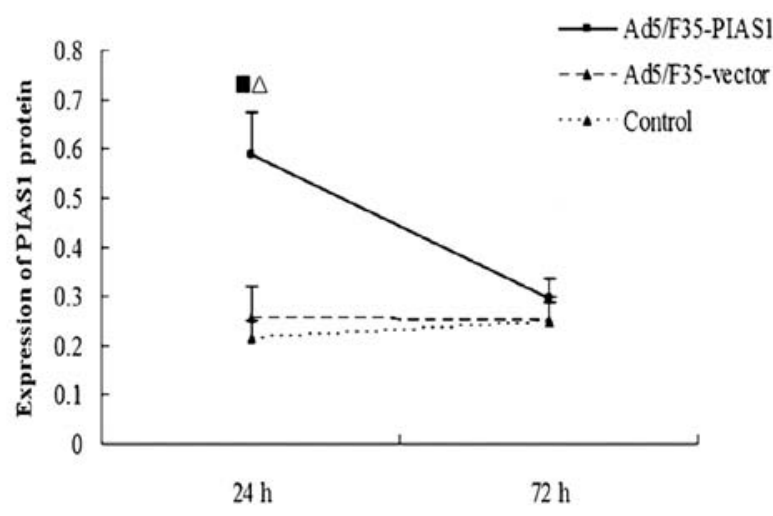

B-2

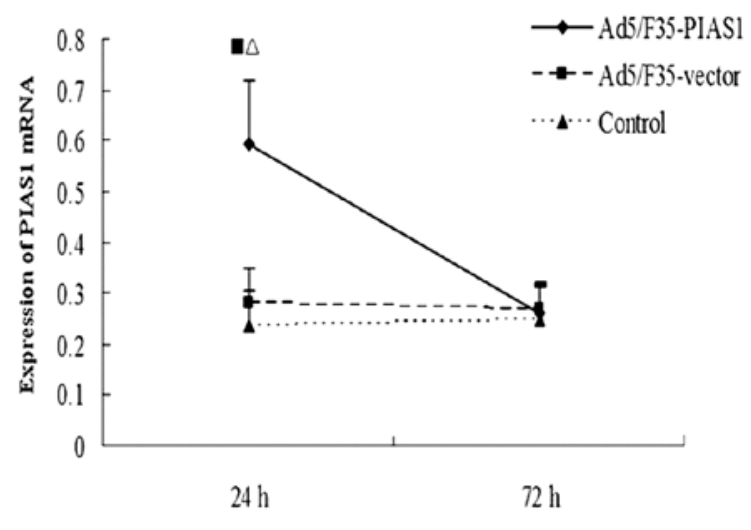

Figure 2. The evaluation of PIAS1 expression was investigated in gastric cancer cell lines SGC7901 in each group. Western blot analysis showing the overexpression of PIAS1 in SGC7901 cells after they had been infected with an adenovirus containing PIAS1 construct in $24 \mathrm{~h}$. The untreated cells and cells infected with adenovirus alone (vector) served as the control that showed lower expression of PIAS1 in $24 \mathrm{~h}$ (A1-2). These results showed similar changes to the results of RT-PCR analysis (B1-2). Note: vs. control group, ${ }^{\wedge} \mathrm{P}<0.01$; vs. Ad5/F35-vector treated group, ${ }^{\mathrm{P}} \mathrm{P}<0.01$. 


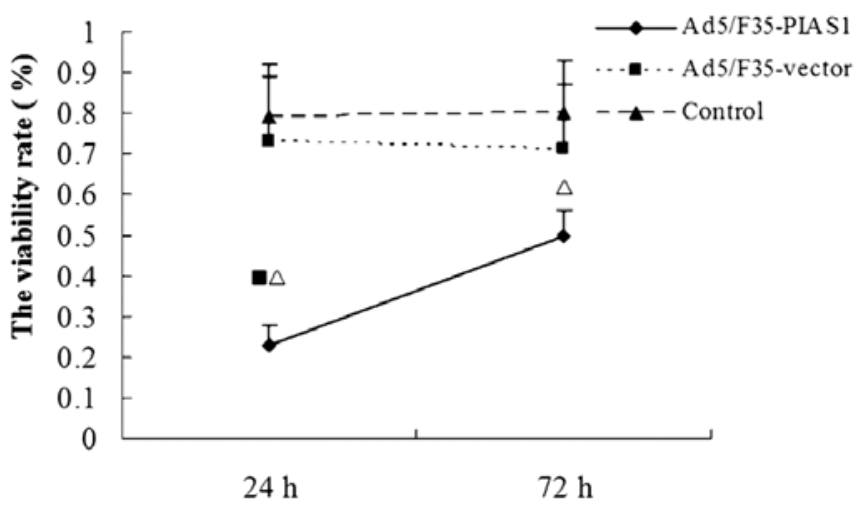

Figure 3. The viability of SGC7901 cells was evaluated by MTT assay in each group. Note: vs. control group, ${ }^{\triangle} \mathrm{P}<0.01$; vs. Ad5/F35-vector treated group, $-\mathrm{P}<0.01$.

\section{A}
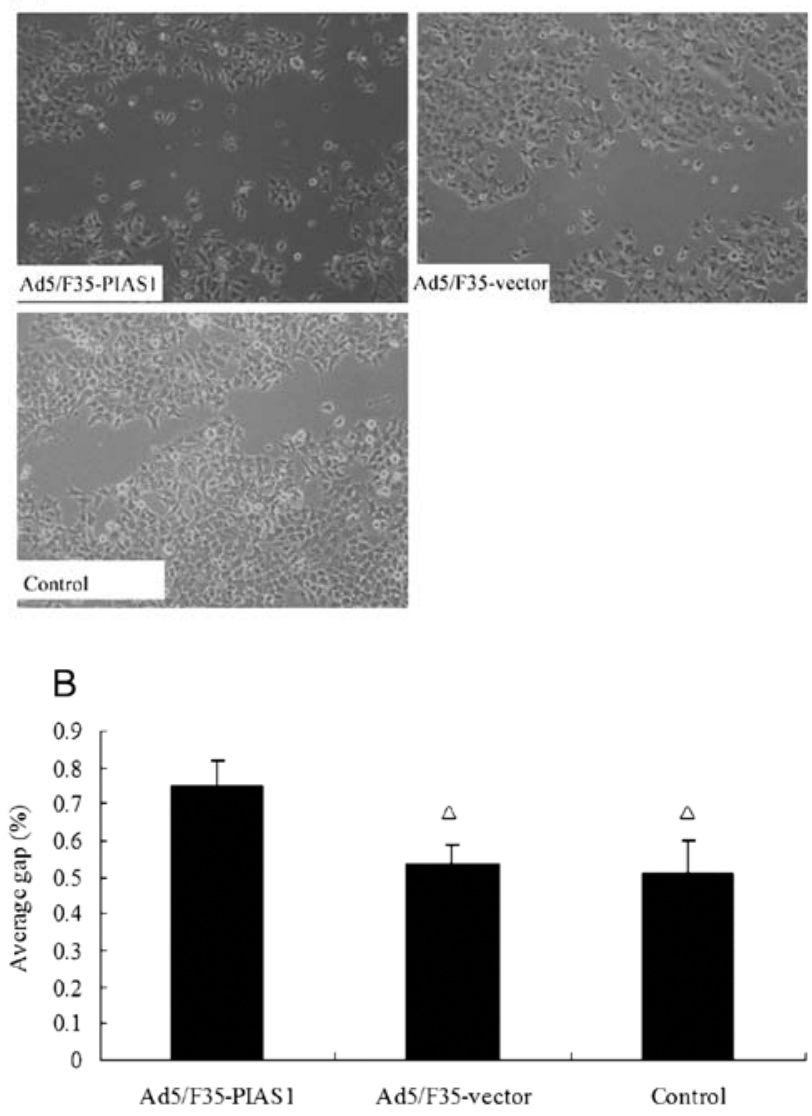

Figure 4. The migration of SGC7901 cells investigated by Scratch woundhealing assay in each group. The cells were infected with control, Ad5/ F35-vector or Ad5/F35-PIAS1 as indicated, respectively. Note: vs. Ad5/F35PIAS1 treated group, ${ }^{\triangle} \mathrm{P}<0.01$.

increase in PIAS1 expression compared with the cells with Ad5/ F35-vector treatment and control.

The proliferation, invasion and migration of gastric cancer cells. We investigated the effect of PIAS1 on proliferation of gastric cancer SGC7901 cells using in vitro MTT assay (Fig. 3). We found that the increased PIAS1 expression by transfection
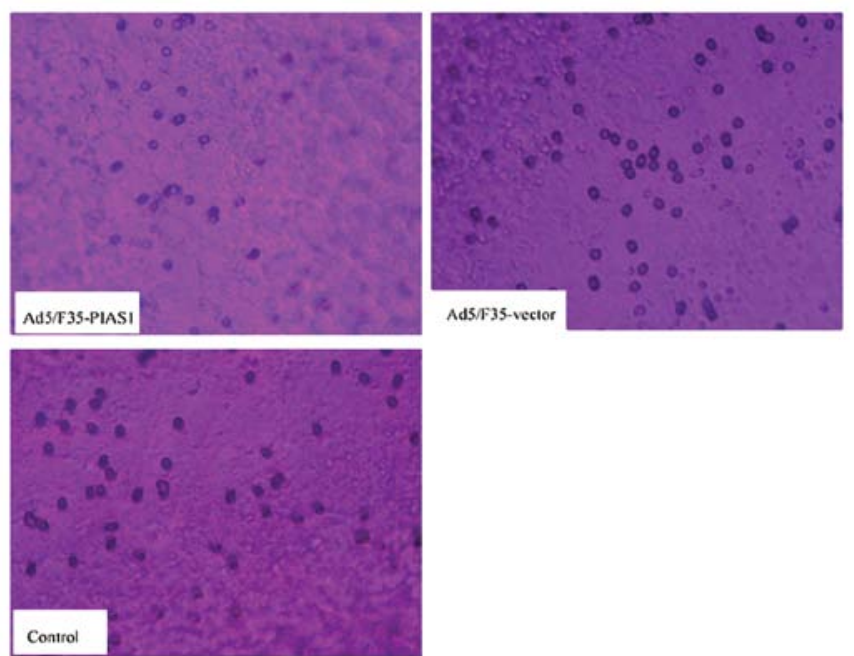

Figure 5. The invasion of SGC7901 cells detected by using Boyden Chamber with Matrigel coating in each group. The cells were infected with control, Ad5/F35-vector or Ad5/F35-PIAS1 as indicated.

with Ad5/F35-PIAS1 significantly decreased the proliferative ability of SGC7901 cells.

To examine the effect of Ad5/F35-PIAS1 on cell motility, an in vitro scratch wound-healing assay was performed (Fig. 4). The results showed that the cells transfected with Ad5/F35-PIAS1 had a reduced migration rate compared with the control cells at $24 \mathrm{~h}(\mathrm{P}<0.01)$. To further investigate the effect of Ad5/F35-PIAS1 on cell invasion, we determined the invasion ability of the three groups of cells using the transwell chambers assay (Fig. 5). After incubation for $24 \mathrm{~h}$, the number of control group and Ad5/F35-PIAS1-vector treated cells which had invaded the polycarbonate membrane of the matrigel chamber was $~ 3.7$ - and 3.3-fold greater than that of the Ad5/F35-PIAS1 treated group, respectively [(21.25 \pm 1.51$)$ and $(19.35 \pm 1.33)$ vs $(5.70 \pm 1.56)](\mathrm{P}<0.05)$. These results provide strong evidence that PIAS1 plays a role in decreasing metastasis of gastric cancer cells.

Expression of P38MAPK, P-P38MAPK, JNK/SAPK, P-JNK/ SAPK, ERK, P-ERK, MMP-9, MMP-2, and ICAM-1 in gastric cancer cells. The proteins of P38MAPK, P-P38MAPK, JNK/ SAPK, P-JNK/SAPK, ERK, and P-ERK were expressed in each group by western blot analysis (Fig. 6A and B). The expression levels of P38MAPK, P-P38MAPK, ERK, and P-ERK proteins were decreased in gastric cancer cells SGC7901 with Ad5/ F35-PIAS1 treatment compared with these cells by transfection with Ad5/F35-vector and control cells. The expression levels of JNK/SAPK, P-JNK/SAPK proteins in gastric cancer with control had no different when compared with those of Ad5/ F35-PIAS1 and Ad5/F35-vector treated cells, respectively. After Ad5/F35-PIAS1 treatment, the expressions of MMP-9, MMP-2, and ICAM-1 were detected in gastric cancers cells, which were decreased compared with those of cells with Ad5/ F35-vector treatment and control, respectively. The average protein levels of MMP-9, MMP-2, and ICAM-1 in gastric cancer cells with control were no higher than those of $\mathrm{Ad} 5 /$ F35-vector treated cells (Fig. 6A and C). 

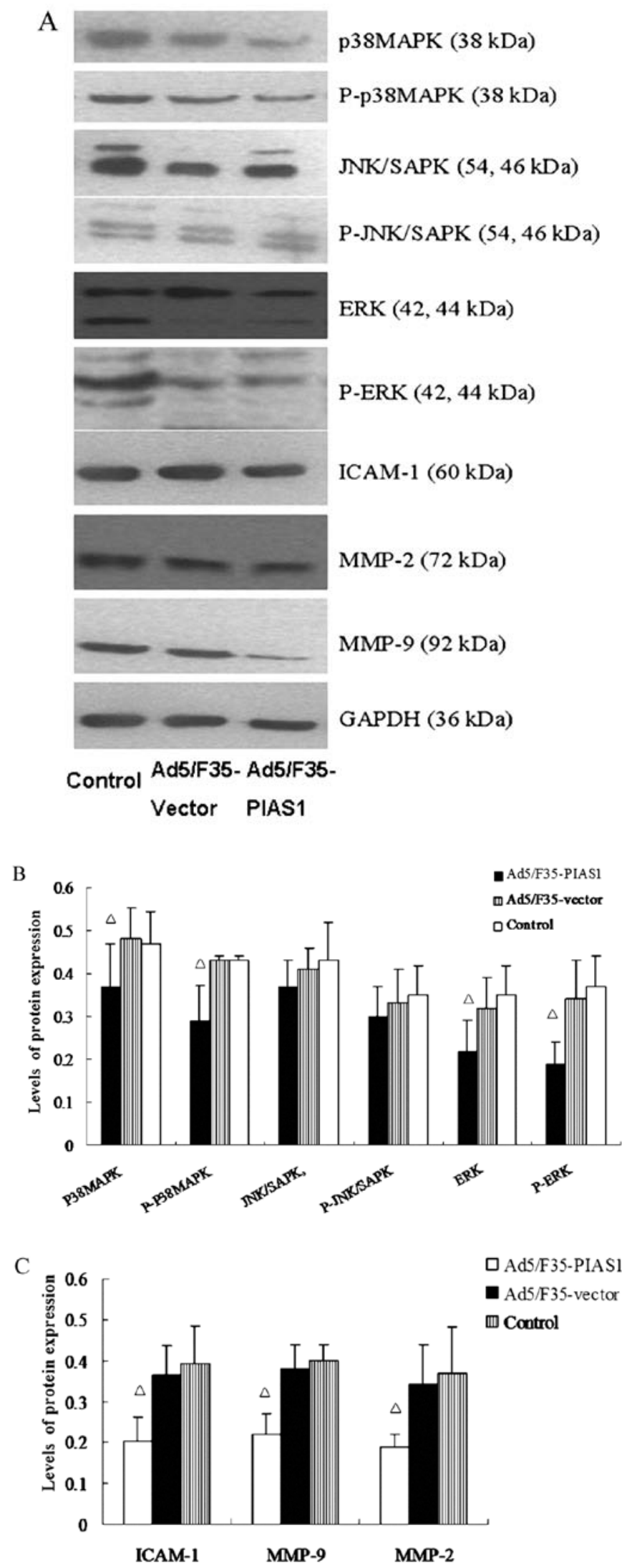

Figure 6. The effective mechanisms of PIAS1 overexpression involved in cell metastasis were investigated by Western blot analysis (A). The MAPK signaling pathway in gastric cancer cells were investigated in SGC7901 cells incubated with control, Ad5/F35-vector, and Ad5/F35-PIAS1, respectively (B). The expression levels of proteins involved in MMP-9, MMP-2, and ICAM-1 were investigated in SGC7901 cells incubated with control, Ad5/ F35-vector, Ad5/F35-PIAS1, respectively (C). Note: vs. control and Ad5/F35vector treated groups, ${ }^{\triangle} \mathrm{P}<0.01$.

\section{Discussion}

Gastric cancer is the most frequent malignancy of the gastrointestinal tract in Chinese populations and the second most common cause of cancer related death in the world. The prognosis of gastric cancer has been improving owing to progress in diagnostic techniques and treatment methods for gastric cancer, but the prognosis of gastric cancer which has invaded is still poor with a 5 -year survival of $<35 \%$ (9). Among these malignant characteristics of gastric cancer cells, metastasis is an especially complex phenomenon, which requires the involvement of many different genes in multiple steps. The adhesion molecules, metastatic chemotaxis related genes, and others have been reported to play an important role in metastasis of gastric cancers $(10,11)$, although many aspects of gastric cancer metastasis await further clarification.

Gene activation analyses show that PIAS1 selectively regulates a subset of STAT dependent genes, with a notable preference for inflammatory cytokines $(11,12)$. In this study, we aimed to investigated the expression of PIAS1 on gastric cancer and evaluated its relationship with development of gastric cancer. Further the study elucidated the upregulating effect of PIAS1 on gastric cancer by a series of studies including cell viability assay and cell invasion, as well as the effective mechanisms of PIAS1, in order to explore a new therapeutic agent for gastric cancer. The PIAS1 expression was significantly decreased in human gastric cancer, which was positively correlated with the development of gastric cancer, but which showed no correlation with tumor size, gender or age with patients. Our results demonstrate that the decreasing expression of PIAS1 was found in TNM stage III and IV tumors. PIAS1 might play an important role in a certain stage. The exact role of PIAS1 in gastric cancer has not been identified. Its upstream and downstream molecular targets are also unidentified. Future investigation will provide the necessary information to shed lights on these points.

Recently, the increasing evidence indicates that anticancer drugs activated many pro-inflammatory signal pathways, some of which were connected to the development of metastasis of tumor cells. The MAPK signaling is an important signaling transduction pathway activated by many different stimuli. Previous reports have shown that the modulators of MAPK signaling pathway can affect cell growth $(13,14)$. With regard to human gastric carcinoma, the investigations of alterations in expression and activity of components of the MAPK cascade will help to understand the mechanisms of malignant behaviors of gastric cancer cells. The P38MAPK is a member of MAPK signaling pathway, mediating many cell reactions induced by stress, inflammatory cytokines or bacterial products and playing a key role in the regulation of cell cycle. For the different cell lines of gastric cell (15), the similar phenomenon can be seen when these cells are presented under P38MAPK activity. Therefore, P38MAPK pathway may be one candidate target of cancer therapy for further study. The activation of signal transduction pathways by growth factors, hormones and neurotransmitters is mediated through JNK/SAPK signaling pathway. It is the basic signal transduction pathway regulating cell proliferation and differentiation (16). Recent research data indicate that the activation of ERK cascade is involved in $H$. pylori-induced proliferation of gastric epithelial cells (17). 
Thus, these results suggest that the MAPK signaling pathway is involved in regulation of the tumor migratory factors in cancer cells, and may contribute to cell growth and cell invasion. Furthermore, this study investigated the relationship of the MAPK signaling pathway and PIAS1 overexpression in gastric cancer. The results of the study showed that the incubation with Ad5/F35-PIAS1 for $24 \mathrm{~h}$ in cells could block ERK and P38MAPK proteins activity. We used Ad5/F35-vector to detect the MAPKS signaling pathway in whole gastric cancer cells, which showed that the administration of Ad5/ F35-vector had no effect on the expression and activation of MAPK signaling pathway including ERK, JNK/SAPK, and P38MAPK proteins.

Local invasion and metastasis are hallmark features in cancer progression. The modulation of the adhesive and migratory properties of the disseminating tumor cells play an essential role in the process of invasion and metastasis. The ability of tumor cells to adhere and migrate across the endothelium and extracellular matrix (ECM) is considered one of the most important prerequisites for the formation of invasion and metastasis (18). ICAM-1 has been found overexpress in gastric cancer and it is correlated with cancer progression (19). The MMPs constitute a large family of endopeptidases, which are responsible for degrading almost all ECM components, with each ECM element being cleaved by a specific MMP. Consistent with their role in tumor progression, the high levels of MMP-2 and MMP-9 have been shown to correlate with poor prognosis (20). We took this experiments further by examining whether MMP-2, MMP-9 and ICAM-1 regulate the invasion of gastric cancer. We further observed the administration of Ad5/F35-PIAS1 in gastric cancer cells markedly decreased the levels of MMP-2, MMP-9 and ICAM-1 proteins. One of the mechanisms by which P38 MAPK may promote tumor cell migration and invasion is by the upregulation of MMPs. Our studies have also demonstrated that MMP-2, MMP-9 and ICAM-1 can directly stimulate cell invasion and invasion via MAPK-dependent mechanism. The low MMP-2, MMP-9 and ICAM-1 protein expression was shown in SGC7901 cells with Ad5/F35-PIAS1 treatment. Therefore, these findings provide a possible explanation how the upregulation of PIAS1 expression could inhibit the invasion of gastric cancer.

In conclusion, the downregulation of PIAS1 expression shows potential to be used as a valuable assessment marker for the progression of gastric cancer. The upregulation of PIAS1 expression inhibited the activation of P38MAPK and ERK proteins and downregulated the levels of downstream cytokines (MMP-2, MMP-9 and ICAM-1), relieving cell invasion. It might be helpful to use PIAS1 in gastric cancer for therapeutic strategy.

\section{References}

1. Bornschein J, Rokkas T, Selgrad M, et al: Gastric cancer: clinical aspects, epidemiology and molecular background. Helicobacter, 16 (Suppl 1): 45-52, 2011.

2. Liu B, Yang R, Wong KA, et al: Negative regulation of NF-kappaB signaling by PIAS1. Mol Cell Biol 25: 1113-1123, 2005.

3. Tahk S, Liu B, Chernishof V, et al: Control of specificity and magnitude of NF-kappa B and STAT1-mediated gene activation through PIASy and PIAS1 cooperation. Proc Natl Acad Sci USA 104: 11643-11648, 2007.

4. Coppola D, Parikh V, Boulware D, et al: Substantially reduced expression of PIAS1 is associated with colon cancer development. J Cancer Res Clin Oncol 135: 1287-1291, 2009.

5. Seger R and Krebs EG: The MAPK signaling cascade. FASEB J 9: 726-735, 1995

6. Whitmarsh AJ and Davis RJ: Transcription factor AP-1 regulation by mitogen-activated protein kinase signal transduction pathways. J Mol Med 74: 589-607, 1996.

7. Graziosi L, Mencarelli A, Santorelli C, et al: Mechanistic role of p38 MAPK in gastric cancer dissemination in a rodent model peritoneal metastasis. Eur J Pharmacol 674: 143-152, 2012.

8. Huang J, Yao WY, Zhu Q, et al: XAF1 as a prognostic biomarker and therapeutic target in pancreatic cancer. Cancer Sci 101: 559-567, 2010.

9. Liu J and Chen L: Current status and progress in gastric cancer with liver metastasis. Chin Med J 124: 445-456, 2011.

10. Tian MM, Sun Y, Li ZW, et al: Polymorphisms of ICAM-1 are associated with gastric cancer risk and prognosis. World $\mathbf{J}$ Gastroenterol 18: 368-374, 2012.

11. Jang BG and Kim WH: Molecular pathology of gastric carcinoma. Pathobiology 78: 302-310, 2011.

12. Tai DJ, Hsu WL, Liu YC, et al: Novel role and mechanism of protein inhibitor of activated STAT1 in spatial learning. EMBO J 30: 205-220, 2011.

13. Liu LM, Yan MG, Yang DH, et al: The expression of protein inhibitor of activated signal transducers and activators of transcription 3 in the evolutionary process of gastric cancer. Eur $\mathbf{J}$ Intern Med 22: e31-35, 2011.

14. Dhillon AS, Hagan S, Rath O, et al: MAP kinase signalling pathways in cancer. Oncogene 26: 3279-3290, 2007.

15. Yang JJ, Cho LY, Ma SH, et al: Oncogenic CagA promotes gastric cancer risk via activating ERK signaling pathways: a nested case-control study. PLoS One 6: e21155, 2011.

16. Wang H, Sun Y, Liu S, et al: Upregulation of progranulin by Helicobacter pylori in human gastric epithelial cells via p38MAPK and MEK1/2 signaling pathway: role in epithelial cell proliferation and migration. FEMS Immunol Med Microbiol 63: 82-92, 2011.

17. Mitsuno Y, Yoshida H, Maeda S, et al: Helicobacter pylori induced transactivation of SRE and AP-1 through the ERK signalling pathway in gastric cancer cells. Gut 49: 18-22, 2001.

18. Matsuoka T, Adair JE, Lih FB, et al: Elevated dietary linoleic acid increases gastric carcinoma cell invasion and metastasis in mice. Br J Cancer 103: 1182-1191, 2010.

19. Haier J, Nasralla M and Nicolson GL: Cell surface molecules and their prognostic values in assessing colorectal carcinomas. Ann Surg 231: 11-24, 2000.

20. Yashiro M, Sunami T and Hirakawa K: CD54 expression is predictive for lymphatic spread in human gastric carcinoma. Dig Dis Sci 50: 2224-2230, 2005.

21. Hara I, Miyake H, Hara S, et al: Significance of matrix metalloproteinases and tissue inhibitors of metalloproteinase expression in the recurrence of superficial transitional cell carcinoma of the bladder. J Urol 165: 1769-1772, 2001. 